\title{
ON GENERALIZING ALTERNATIVE RINGS
}

\section{J. RODABAUGH}

ABSTRACT. Consider a ring $R$ that satisfies the identity $(x, x, x)$ $=0$ and any two of the three identities: $(w x, y, z)+(w, x,[y, z])-$ $w(x, y, z)-(w, y, z) x=0 ;([w, x], y, z)+(w, x, y z)-y(w, x, z)-$ $(w, x, y) z=0 ;(w, x \cdot y, z)-x \cdot(w, y, z)-y \cdot(w, x, z)=0$. In this paper, we prove that if $R$ has characteristic prime to 6 then $R$ semiprime with idempotent $e$ implies $R$ has a Peirce decomposition in which the modules multiply as they do in an alternative ring. If in addition $R$ is prime with idempotent $e \neq 0,1$ then $R$ is alternative.

1. Introduction. Let $R$ be a nonassociative ring. For $x, y, z$ in $R$ define the associator $(x, y, z)=(x y) z-x(y z)$ and the commutator $[x, y]=$ $x y-y x$. The ring $R$ is power-associative if for each $x$ in $R$, the subring generated by $x$ is associative.

In this paper we will be concemed with rings that satisfy the identity

$$
(x, x, x)=0
$$

and any two of the three identities:

$$
\begin{aligned}
& g(w, x, y, z)=(w x, y, z)+(w, x,[y, z])-w(x, y, z)-(w, y, z) x=0 \\
& h(w, x, y, z)=(w, x \cdot y, z)-x \cdot(w, y, z)-y \cdot(w, x, z)=0 \\
& k(w, x, y, z)=([w, x], y, z)+(w, x, y z)-y(w, x, z)-(w, x, y) z=0 .
\end{aligned}
$$

Rings satisfying (1), (2) and (4) were studied by Kleinfeld [2] and Smith [3]. Rings satisfying (1), (2) and (3) are anti-isomorphic to rings which satisfy (1), (3) and (4). This paper will concentrate on rings satisfying (1), (2) and (3). By anti-isomorphism our results will hold for rings satisfying (1), (3) and (4). These results are known for rings satisfying (1), (2) and (4) (see [3]).

From now on we assume $R$ to be a ring satisfying (1), (2) and (3). It

Received by the editors September 17, 1973.

AMS (MOS) subject classifications (1970). Primary 17A30.

Key words and phrases. Alternative ring, prime ring, semiprime ring. 
is known [1] that $R$ is power-associative. For characteristic prime to 2 we define $x \cdot y=1 / 2(x y+y x)$. If $R$ contains an idempotent then

$$
R=R_{1}(e)+R_{1 / 2}(e)+R_{0}(e)
$$

where $R_{i}(e)=\{x: x \cdot e=i x\}$. This is the Albert decomposition of $R$. We will denote $R_{i}(e)$ by $R_{i}$.

A nonassociative ring $R$ is semiprime if $R$ contains no nonzero ideal $I$ such that $I^{2}=0$. The ring $R$ is prime if $R$ contains no nonzero ideals $I$ and $H$ such that $I H=0$.

Theorem 1. The following are equivalent for any nonassociative ring:

(a) $R$ is prime.

(b) $R$ contains no nonzero ideals $I$ and $H$ such that $I H+H I=0$.

(c) $R$ contains no nonzero ideals $I$ and $H$ such that $H I=0$.

Proof. If $R$ contains nonzero ideals $I$ and $H$ with $I H+H I=0$ then $\dot{I H}=0$ so $R$ is not prime. Thus (a) implies (b). To prove (b) implies (a) assume $I, H$ are nonzero ideals with $I H=0$ and assume $R$ satisfies (b). Then $H I=I H+H I \neq 0$. Let $K=H \cap I$. We have $H I \subseteq K$ so $K \neq 0$. However, $K^{2} \subseteq I H=0$ so $K=0$ a contradiction. We have proved (a) equivalent to (b). The proof that (b) and (c) are equivalent follows by symmetry.

Theorem 2. Let $R$ be semiprime. Then $R$ is prime if and only if $R$ contains no nonzero ideals $I$ and $H$ with $I \cap H=0$.

Proof. If $I \cap H=0$ then $I H \subseteq I \cap H=0$. If $I H=0$, define $K=I \cap H$. We have $K^{2} \subseteq I H=0$ so $K=0$.

2. Preliminaries. We assume throughout that $R$ satisfies (1), (2) and (3) with characteristic prime to 6 . The following identity holds in any ring.

$$
t(w, x, y, z)=(w x, y, z)-(w, x y, z)+(w, x, y z)-w(x, y, z)-(w, x, y) z=0 .
$$

If we subtract $g(w, x, y, z)-t(w, x, y, z)=0$ we obtain

$$
s(w, x, y, z)=(w, x y, z)-(w, x, z y)+(w, x, y) z-(w, y, z) x=0 .
$$

We also note

$$
g(w, x, y, y)=(w x, y, y)-w(x, y, y)-(w, y, y) x=0
$$

From [1] we know that if $e$ is an idempotent then $(e, R, e)=0$. In the Albert decomposition $R_{i} R_{1 / 2}+R_{1 / 2} R_{i} \subseteq R_{1 / 2}$ for $i=0$, 1. Defining $B$ by $B=$ 
\{x in $\left.R_{1 / 2}: x R+R x \subseteq R_{1 / 2}\right\}$ it is known [1] that $B$ is an ideal in which each element squares to zero.

If $R$ has a Peirce decomposition $R=R_{11}+R_{10}+R_{01}+R_{00}\left(R_{i j}=\right.$ $\{x: e x=i x, x e=j x\}$ ), then $R_{i j} R_{k m} \subseteq \delta_{j k} R_{i m}$ (Kronecker delta) with the following exceptions: $R_{i j} R_{i j} \subseteq R_{j i}{ }^{\circ}(i \neq j) ; R_{i i} R_{j i} \subseteq R_{i j}$. This latter exception is the most pathological. However, if we define

$$
J=\left(R_{11} R_{01}\right) R_{01}+R_{11} R_{01}+R_{00} R_{10}+\left(R_{00} R_{10}\right) R_{10}
$$

then $J$ is an ideal and $J^{2}=0$ (see [1])。

\section{Semiprime rings.}

Lemma 3. Under our hypotheses, the following hold:

(a) $\left(R_{1 / 2}^{2}, e, e\right)=\left(e, e, R_{1 / 2}^{2}\right)=0$.

(b) $([R, R], e, e)=(e, e,[R, R])=0$.

(c) $(e, R, R),(R, e, R),(R, e, e)$ are in $R_{1 / 2}$.

(d) $(e, e, R)$ and $(R, e, e)$ are in $B$.

Proof. We first note the following linearizations of (1):

$$
(x, y, z)+(y, z, x)+(z, x, y)+(z, y, x)+(y, x, z)+(x, z, y)=0 .
$$

$$
(x, y, y)+(y, y, x)+(y, x, y)=0
$$

Now (c) and (d) were proved in [1]. From (7) we have

$$
g(e, e, y, z)=(e, y, z)+(e, e,[y, z])-e(e, y, z)-(e, y, z) e=0 .
$$

By $(c),(e, y, z)=e(e, y, z)+(e, y, z) e$ so $(e, e,[y, z])=0$. Since $(e, R, e)$ $=0,(e,[R, R], e)=0$ so (9) implies $([y, z], e, e)=0$ and (b) is established. Let $x$ and $y$ be in $R_{1 / 2}$. Clearly $x y=x \cdot y+1 / 2[x, y]$. It is known [1] that $x \cdot y$ is in $R_{1}+R_{0}$ so $(x \cdot y, e, e)=(e, e, x \cdot y)=0$. Thus

$$
(x y, e, e)=(x \cdot y, e, e)+1 / 2([x, y], e, e)=0 \text {. }
$$

By symmetry, $(e, e, x y)=0$ and (a) is proved.

Theorem 4. Let $I=(R, e, e)$. Then $I$ is an ideal with $[R, I]=I^{2}=R_{1 / 2} I$ $=0$.

Proof. Let $I^{\prime}=(e, e, R)$. From (9) and the fact that $(e, R, e)=0$ we have $I=I^{\prime}$. Now, let $b=(x, e, e)$. From Lemma $3(\mathrm{~d}), b \in B$ so $b e+e b=b$. We note, from (5) and the fact that $(e, R, e)=0$, 


$$
\begin{aligned}
0 & =t(e, e, x, e) \\
& =(e, x, e)-(e, e x, e)+(e, e, x e)-e(e, x, e)-(e, e, x) e \\
& =(e, e, x e)-(e, e, x) e .
\end{aligned}
$$

Substituting $w=x=y=e$ and $z=x$ in (6) yields

$$
\begin{aligned}
0 & =s(e, e, e, x) \\
& =(e, e, x)-(e, e, x e)+(e, e, e) x-(e, e, x) e \\
& =(e, e, x)-(e, e, x e)-(e, e, x) e .
\end{aligned}
$$

If we add (10) and (11) we have $(e, e, x)=2(e, e, x) e$. Since $b$ is in $I^{\prime}$ for $I=I^{\prime}$ this means $b=2 b e$ so $b e=e b=1 / 2 b$. We now choose $b$ and $b^{\prime}$ from $I \subseteq B \subseteq R_{1 / 2}$. Lemma $3(a)$ implies $\left(b b^{\prime}, e, e\right)=0$ so

$$
\begin{aligned}
0 & =g\left(b, b^{\prime}, e, e\right) \\
& =\left(b b^{\prime}, e, e\right)+\left(b, b^{\prime},[e, e]\right)-b\left(b^{\prime}, e, e\right)-(b, e, e) b^{\prime} \\
& =-b\left(b^{\prime}, e, e\right)-(b, e, e) b^{\prime} .
\end{aligned}
$$

Since, for $b$ in $I, b e=e b=1 / 2 b,(b, e, e)=1 / 2 b e-1 / 2 b=1 / 4 b-1 / 2 b=-1 / 4 b$.

Therefore, from (12), $0=-b\left(b^{\prime}, e, e\right)-(b, e, e) b^{\prime}=1 / 4 b b^{\prime}+1 / 4 b b^{\prime}=1 / 2 \cdot b b^{\prime}$. We have proved $I^{2}=0$.

Define $K=(I, e, e)$. As above we easily see $K=(e, e, I)$. Let $b=$ $(x, e, e)$. Then $(b, e, e)=-1 / 4 b$ as above so $b$ is in $K$. Therefore $K=I$ and we have proved $I=(I, e, e)=(e, e, I)$. From (7) we have the important fact

$$
(w x, e, e)=w(x, e, e)+(w, e, e) x \text {. }
$$

If $w$ is in $R_{1}+R_{0}$ then $(w, e, e)=0$ and $w(x, e, e)$ is in $I$. Therefore $\left(R_{1}+R_{0}\right) I \subseteq I$. Similarly $I\left(R_{1}+R_{0}\right) \subseteq I$. Now let $w$ be in $R_{1 / 2}$. By Lemma $3(\mathrm{a}),(w(x, e, e), e, e)=0$ since $(x, e, e)$ is in $I \subseteq R_{1 / 2}$. Since $I^{2}=0$, $(w, e, e)(x, e, e)=0$ so from (13) we have $w((x, e, e), e, e)=0$. But, if $b=(x, e, e)$ is in $I$ then $w(b, e, e)=-1 / 4 w b$ so $w b=0$ and $R_{1 / 2} I=0$. Similarly $I R_{1 / 2}=0$. Thus, $I$ is an ideal of $R$ and $R_{1 / 2} I=0$.

To see that $[R, I]=0$ we first note that $\left[R_{1 / 2}, I\right]=0$ was proved above. If $w$ is in $R_{1}+R_{0}$ then $(w, e, e)=0$. By (13), $(w x, \dot{e}, e)=w(x, e, e)$ and $(x w, e, e)=(x, e, e) w$. From Lemma $3(\mathrm{~b})$ we have $0=([w, x], e, e)=$ $[w,(x, e, e)]$ so $[R, I]=0$.

Theorem 5. Let $R$ satisfy (1), (2) and (3) with characteristic prime to 6. If $e$ is an idempotent in $R$ and if $R$ is semiprime then $R$ has a Peirce decomposition relative to $e$. Furthermore the modules $R_{i j}$ multiply as in an alternative ring $\left(R_{i j} R_{k m} \subseteq \delta_{j k} R_{i m}\right.$ except $R_{i j}^{2} \subseteq R_{j i}$ for $\left.i \neq j\right)$ and $T=$ $R_{10} R_{01}+R_{10}+R_{01}+R_{01} R_{10}$ is an alternative ideal. 
Proof. From Theorem 4 and its proof, $I=(R, e, e)=(e, e, R)$ is such that $I^{2}=0$ and $I$ is an ideal. Therefore $I=0$. Since $(e, R, e)=0$ then $R$ has a Peirce decomposition. Also by semiprimeness, the ideal $J$ defined in $\S 2$ is zero so $R_{i j} R_{k m} \subseteq \delta_{j k} R_{i m}$ except that for $i \neq j R_{i j}^{2} \subseteq R_{j i}$. The arguments in [1] now show that $T$ is an alternative ideal.

\section{Prime rings.}

Lemma 6. If $R$ is semiprime then $\left(R_{j i}, R_{i i}, R_{i i}\right)=\left(R_{i i}, R_{i i}, R_{i j}\right)=0$ for $i \neq j$.

Proof. We will use letters with subscripts to denote elements in $R_{i j}$, etc. For example, $w_{10}$ in $R_{10}$. We compute

$$
\begin{aligned}
0 & =b\left(w_{01}, e, y_{11}, z_{11}\right) \\
& =\left(w_{01}, e \cdot y_{11}, z_{11}\right)-e \cdot\left(w_{01}, y_{11}, z_{11}\right)-y_{11} \cdot\left(w_{01}, e, z_{11}\right) .
\end{aligned}
$$

However, $\left(w_{01}, e, z_{11}\right)=0,\left(w_{01}, y_{11}, z_{11}\right)$ is in $R_{01}$. Therefore, $0=$ $\left(w_{01}, y_{11}, z_{11}\right)-1 / 2\left(w_{01}, y_{11}, z_{11}\right)=1 / 2\left(w_{01}, y_{11}, z_{11}\right)$. Again using (3), we obtain

$$
\begin{aligned}
0 & =h\left(w_{10}, e, y_{00}, z_{00}\right) \\
& =\left(w_{10}, e \cdot y_{00}, z_{00}\right)-e \cdot\left(w_{10}, y_{00}, z_{00}\right)-y_{00} \cdot\left(w_{10}, e, z_{00}\right) \\
& =-1 / 2\left(w_{10}, y_{00}, z_{00}\right) .
\end{aligned}
$$

Therefore $\left(R_{j i}, R_{i i}, R_{i i}\right)=0$ for $i \neq j$. Using $0=h\left(z_{11}, y_{11}, e, w_{10}\right)=$ $h\left(z_{00}, y_{00}, e, w_{01}\right)$ we can similarly prove $\left(R_{i i}, R_{i i}, R_{i j}\right)=0$ for $i \neq j$.

Definition. Define $P_{i}=\left(R_{i i}, R_{i i}, R_{i i}\right)$ and $S_{i}=P_{i}+P_{i} R_{i i}$ for $i=$ 0,1 .

Lemma 7. If $R$ is semiprime then $R_{i j} S_{i}=S_{i} R_{j i}=R_{j j} S_{i}=S_{i} R_{j j}=$ $R_{j i} S_{i}=S_{i} R_{i j}=0$ for $i \neq j$.

Proof. Since $S_{i} \subseteq R_{i i}, R_{i j} S_{i}=S_{i} R_{j i}=R_{j j} S_{i}=S_{i} R_{j j}=0$ follows from the multiplication of the modules $R_{i j}$. Compute

$$
\begin{aligned}
0 & =g\left(w_{11}, x_{10}, y_{11}, z_{11}\right) \\
& =\left(w_{11} x_{10}, y_{11}, z_{11}\right)+\left(w_{11}, x_{10},\left[y_{11}, z_{11}\right]\right)-w_{11}\left(x_{10}, y_{11}, z_{11}\right)-\left(w_{11}, y_{11}, z_{11}\right) x_{10} \\
& =-\left(w_{11}, y_{11}, z_{11}\right) x_{10}
\end{aligned}
$$

since $R_{10} R_{11}=0$. Therefore $P_{1} R_{10}=0$. From $0=g\left(w_{00}, x_{01}, y_{00}, z_{00}\right)$ we show similarly that $P_{0} R_{01}=0$. Applying Lemma 6 we have for $i \neq j$, $S_{i} R_{i j}=P_{i} R_{i j}+\left(P_{i} R_{i i}\right) R_{i j}=P_{i}\left(R_{i i} R_{i j}\right) \subseteq P_{i} R_{i j}=0$. 
To prove $R_{01} P_{1}=0$, write

$$
\begin{aligned}
0 & =b\left(w_{11}, x_{01}, y_{11}, z_{11}\right) \\
& =\left(w_{11}, x_{01} \cdot y_{11}, z_{11}\right)-x_{01} \cdot\left(w_{11}, y_{11}, z_{11}\right)-y_{11} \cdot\left(w_{11}, x_{01}, z_{11}\right) \\
& =-1 / 2 x_{01}\left(w_{11}, y_{11}, z_{11}\right)
\end{aligned}
$$

since $R_{11} R_{01}=0$. Also, $0=h\left(w_{00}, x_{10}, y_{00}, z_{00}\right)$ implies $R_{10} P_{0}=0$. By Lemma 6 we have for $i \neq j, R_{i j} S_{j}=R_{i j} P_{j}+R_{i j}\left(P_{j} R_{j j}\right)=\left(R_{i j} P_{j}\right) R_{j j}=0$.

Theorem 8. If $R$ is a semiprime ring satisfying (1), (2), and (3) with characteristic prime to 6 then $S_{1}$ and $S_{0}$ are ideals. In addition, $S_{1} S_{0}=$ $T S_{1}=\left(T S_{0}\right) R_{00}=0$.

Proof. We first prove that each $S_{i}$ is an ideal. From Lemma 7, all that is needed is to show that $S_{i} R_{i i}+R_{i i} S_{i} \subseteq S_{i}$. Clearly, $S_{i} R_{i i}=P_{i} R_{i i}+$ $\left(P_{i} R_{i i}\right) R_{i i} \subseteq P_{i} R_{i i}$ (by Lemma 6) $\subseteq S_{i}$. Using (2), we have

$$
\begin{aligned}
0 & =g\left(w_{i i}, x_{i i}, y_{i i}, z_{i i}\right) \\
& =\left(w_{i i} x_{i i}, y_{i i}, z_{i i}\right)+\left(w_{i i}, x_{i i},\left[y_{i i}, z_{i i}\right]\right)-w_{i i}\left(x_{i i}, y_{i i}, z_{i i}\right)-\left(w_{i i}, y_{i i}, z_{i i}\right) x_{i i} \cdot
\end{aligned}
$$

Define $D_{i}=P_{i}+R_{i i} P_{i}$ for $i=0,1$. By (14), $D_{i} \subseteq S_{i}$ and $S_{i} \subseteq D_{i}$ so $D_{i}=$ $S_{i}$. Therefore $R_{i i} S_{i}=R_{i i} D_{i}=R_{i i} P_{i}+R_{i i}\left(R_{i i} P_{i}\right) \subseteq R_{i i} P_{i}$ (by Lemma 6) $\subseteq D_{i}=S_{i}$

To see that $S_{1} S_{0}=0$ we need only note that $S_{1} S_{0} \subseteq R_{11} R_{00}=0$.

Before proceeding we must prove that $\left(R_{i j}, R_{j i}, R_{i i}\right) R_{i i}=0$ for $i \neq j$. We have

$$
\begin{aligned}
0 & =g\left(w_{i j}, x_{i i}, y_{j i}, z_{i i}\right) \\
& =\left(w_{i j} x_{i i}, y_{i i}, z_{i i}\right)+\left(w_{i j}, x_{i i},\left[y_{j i}, z_{i i}\right]\right)-w_{i j}\left(x_{i i}, y_{j i}, z_{i i}\right)-\left(w_{i j}, y_{j i}, z_{i i}\right) x_{i i} \\
& =-\left(w_{i j}, y_{j i}, z_{i i}\right) x_{i i}
\end{aligned}
$$

since $R_{i i} R_{j i}=R_{i j} R_{i i}=0$. Indeed, $\left(w_{10}, y_{01}, z_{11}\right)=\left(w_{10}, y_{01}, z_{11}\right) e=0$. Consequently, $\left(R_{10} R_{01}\right) S_{1}=R_{10}\left(R_{01} S_{1}\right)=0$ by Lemma 7 . Using the definition of $T$ and Lemma 7 we conclude that $T S_{1}=0$. Finally, from $\left(w_{01}, y_{10}, z_{00}\right) x_{00}=0$, the definition of $T$ and Lemma $7,\left(T S_{0}\right) R_{00}=0$.

Theorem 9. If $R$ is a prime ring satisfying (1), (2) and (3) with characteristic prime to 6 and if $R$ contains an idempotent $e \neq 1$ then $R$ is alterna. tive.

Proof. If $T=0$ then $R=R_{11} \oplus R_{00}$ so $R_{00}=0$. and $e$ is the unity 
el ement contrary to assumption. Hence, $T \neq 0$. From Theorem $8, T S_{1}=0$ so $S_{1}=0$. From the definition of $T$ and Lemma 7, $T S_{0} \subseteq\left(R_{01} R_{10}\right) S_{0}$. Define $T_{0}=R_{01} R_{10}$. Now, $S_{0}$ is an ideal so $T S_{0} \subseteq S_{0}$. Therefore, by Lemma 7, $\left(T S_{0}\right) R=\left(T S_{0}\right) R_{00}$ and $R\left(T S_{0}\right)=R_{00}\left(T S_{0}\right)$. Using Theorem $8,\left(T S_{0}\right) R_{00}=0$ so $\left(T S_{0}\right) R=0$. To show $T S_{0}$ is an ideal we need only have $R\left(T S_{0}\right) \subseteq T S_{0}$. But, $R\left(T S_{0}\right)=R_{00}\left(T_{0} S_{0}\right)=R_{00}\left(\left(R_{01} R_{10}\right) S_{0}\right)$. From (3),

$$
\begin{aligned}
0 & =h\left(w_{00}, x_{01}, y_{10}, s_{0}\right) \\
& =\left(w_{00}, x_{01} \cdot y_{10}, s_{0}\right)-x_{01} \cdot\left(w_{00}, y_{10}, s_{0}\right)-y_{10} \cdot\left(w_{00}, x_{01}, s_{0}\right) \\
& =1 / 2\left(w_{00}, x_{01} y_{10}, s_{0}\right) .
\end{aligned}
$$

Therefore $R_{00}\left(T_{0} S_{0}\right)=\left(R_{00} T_{0}\right) S_{0} \subseteq T_{0} S_{0}$ since $T$ is an ideal. Therefore, $T S_{0}$ is an ideal. Another application of Theorem 8 gives $\left(T S_{0}\right) S_{0}=0$ so $T S_{0}=0$ or $S_{0}=0$. But, if $T S_{0}=0$ then $S_{0}=0$ since $T \neq 0$.

We conclude that $S_{1}=S_{0}=0$. Therefore $R_{11}$ and $R_{00}$ are associative and the proof in [1] that $T$ is altemative can be repeated to show that $R$ is alternative. All they needed was that $R_{11}$ and $R_{00}$ be associative.

\section{REFERENCES}

1. Seyoum Getu and D. J. Rodabaugh, Generalizing alternative rings, Communications in Algebra (to appear).

2. E. Kleinfeld, Generalization of alternative rings. I, J. Algebra 18 ( 1971 ), 304-325. MR 43 \# 308.

3. Harry F. Smith, Prime generalized alternative rings I with nontrivial idempotent, Proc. Amer. Math. Soc. 39 (1973), 242-246.

DEP ARTMENT OF MATHEMATICS, UNIVERSITY OF MISSOURI, COLUMBIA, MISSOURI 65201 